\title{
Motor paralysis during asphyxial block of the ulnar nerve
}

\author{
D. C. SinClair, A. B. MATHESON, AND W. G. SKENE \\ From the Department of Anatomy, Aberdeen University
}

SUMMARY During blocks of the ulnar nerve induced by inflating a sphygmomanometer cuff round the arm, the strengths of the movements of ulnar deviation of the individual fingers and adduction of the thumb were measured by means of a pressure transducer. No statistically significant differences were found between the times at which failure of these movements occurred, and there was no evidence of the occurrence of a centripetal motor paralysis within the territory of the ulnar nerve in the hand. However, the flexor carpi ulnaris did not become paralysed until significantly later than the small muscles of the hand. The results do not support the idea that long motor fibres are more susceptible to asphyxia than short ones, at least when the difference in length is $4 \mathrm{~cm}$ or less. Such a theory could certainly explain the relative immunity of the flexor carpi ulnaris, but other explanations for this finding may be available.

In 1931 Lewis, Pickering, and Rothschild investigated the results of applying a sphygmomanometer cuff, at a pressure greater than systolic blood pressure, in various situations on the upper limb. They described a 'centripetal paralysis' of sensation and motor power, and deduced from this that nerve fibres supplying more distal regions are more vulnerable to asphyxia than fibres supplying more proximal regions. They also found that the supply of the extensor muscles of the forearm failed before that of the flexor muscles. These observations were confirmed by Kugelberg (1948a, b), who wrote: 'The longer tactile fibers, the longer motor fibers, the shorter tactile fibers, the extensor motor fibers and the flexor motor fibers are activated and paralysed, in the order of enumeration.'

Sinclair (1948) showed that all the observations made on sensory paralysis in such circumstances could be explained by reference to the anatomy of the upper limb, and that the paralysis was not truly 'centripetal', but depended on the successive involvement of different nerve trunks, probably because pressure was not equally transmitted within the limb. The sensory paralysis within the territory of the ulnar nerve was not centripetal at all.

It has not yet been shown that the motor paralysis can be explained in this way, and there is some electrophysiological evidence that motor fibres may behave differently from sensory fibres during asphyxia. For example, Leksell (1945) found that in a selective pressure block he could eliminate activity in the alpha fibres before the gamma fibres, and this is the reverse of the usual order of failure among sensory fibres (Sinclair, 1967). Groat and Koenig (1946a) clamped the trachea in a cat, and found that asphyxia caused a proximodistal gradient of functional deterioration in a peripheral nerve after the death of the animal. This work was criticized by Frankenhaeuser (1949), who pointed out that differences in operative technique could cause differences in the access of oxygen to different stretches of nerve. However, Groat and Koenig (1946b) described a similar gradient of proximodistal deterioration in the facial nerve inside the nervous system, and this finding requires explanation.

In the present investigation we examined the paralysis of the muscles supplied by the ulnar nerve when the upper limb was asphyxiated by a compression cuff. This nerve was chosen because it supplies in sequence a series of small muscles which are more or less comparable in weight and power: a tendency to centripetal spread of paralysis should therefore be easily detected.

\section{METHODS}

Thirty-three subjects took part, all of whom were medical students or members of the staff of the Department of Anatomy.

A general routine for testing a selection of the muscles supplied by the ulnar nerve has been described by Matheson, Sinclair, and Skene (1970), and was used again in this investigation. A pegboard was mounted 
vertically on the side of a chair; bolted to the board was a pressure transducer connected to an ammeter, on the scale of which the readings increased linearly with increasing pressure. These readings were converted into grams of pressure after calibration of the instrument. The subject sat with his arm hanging down vertically, the finger to be tested lying in line with the transducer, with the base of the fingernail opposite the end of the thrust plate, so that a standard length of lever was applied every time that particular finger was tested. The adjacent fingers were restrained by metal pins fitted into the holes in the board, and the ulnar side of the hand was restrained by a movable metal barrier, so that movements of the wrist could not be transmitted to the transducer. The metacarpals were held flat to the board by pressure of the experimenter's hand.

Each subject underwent a preliminary standard testing session in which he deviated in turn each finger of the left hand in an ulnar direction as hard as he could for a period of three seconds, timed with a metronome; each finger made six such deviations in a total time of six minutes. The thrust of each individual finger was recorded as the mean of the six readings for this finger. In some subjects the power of adduction of the thumb was measured by using another transducer mounted at right angles to the edge of the board.

Asphyxial paralysis was induced by placing a sphygmomanometer cuff as high as possible on the arm, which was first drained of blood by holding it up above the head. The cuff was rapidly pumped up and the arm was then lowered. The pressure was maintained at $180 \mathrm{~mm} \mathrm{Hg}$ for the duration of the experiment, which was timed by a stopwatch. Motor testing (using ulnar deviation of the individual fingers, or adduction of the thumb) did not begin until after signs of sensory failure had become pronounced, usually about 22 minutes after inflation of the cuff (Sinclair, 1948). A quarter of a minute was allowed for positioning and testing each finger, so that a 'round' of tests occupied one minute. The time of complete failure of the finger to deviate-that is, the time at which no movement at all was perceptible on the ammeter-was recorded. In some experiments the time of failure of the flexor carpi ulnaris was investigated; contraction of the muscle was assessed by palpation of its tendon just proximal to the pisiform bone, the subject being asked to flex and adduct his wrist.

\section{RESULTS}

PRELIMINARY EXPERIMENTS Since functional deterio ration of the asphyxiated muscles might be a factors in determining the onset of motor paralysis, it waş decided to limit movements of the fingers to the absolute minimum required to establish the time of failure. For this reason, preliminary trials were undertaken to determine the approximate time of the first muscle failure in each individual.

These preliminary experiments involved 19 subjects. In Group $A$ (seven males and five females) ulnar deviation of all four fingers was used; ino Group B (four males and three females) ulnar devia $\frac{\bar{s}}{\sqrt{3}}$ tion of the little and middle fingers was comparedब with adduction of the thumb.

The results are shown in Table 1 , together with the initial strengths of the movements tested, taken $\overrightarrow{0}$ from the preliminary standard sessions. In Group $A-\overrightarrow{-}$ the index and little fingers were both significantly stronger than the middle and the ring fingers ( $P<0.001$ in all cases), but the differences between the index and little fingers and between the middlecw and ring fingers did not reach the $5 \%$ level of signi ficance. In Group B adduction of the thumb was over ten times as powerful as the movements of theo little and middle fingers $(P<0.001)$. In neither group were there any statistically significant differ ences between the times of failure of the individta $B$ movements tested.

The preliminary experiments revealed two difficin ties which had not been appreciated at the outse Firstly, as the muscles became progressively weak the fingers tended to flex and curl up, making side-:ways deviation awkward. Secondly, it was felt thato the order of testing the movements (which was $\leqslant$ always the same, beginning with the little finger andō ending with the index finger) might possibly intro-مِ duce a systematic bias into the results.

DEFINITIVE EXPERIMENTS Twelve subjects (five males

TABLE 1

PRELIMINARY EXPERIMENTS

\begin{tabular}{|c|c|c|c|c|}
\hline & Little finger & Ring finger & Middle finger & Index finger \\
\hline \multicolumn{5}{|c|}{$\begin{array}{l}\text { Group } A: 12 \text { subjects: ulnar deviation of fingers } \\
\text { Means and standard deviations of: }\end{array}$} \\
\hline \multirow[t]{2}{*}{$\begin{array}{l}\text { times of failure (min) } \\
\text { initial strength of movements }(\mathrm{g})\end{array}$} & $\begin{array}{r}25 \cdot 7 \pm 3 \cdot 0 \\
769 \pm 187\end{array}$ & $\begin{array}{r}25 \cdot 0 \pm 2 \cdot 8 \\
443 \pm 105\end{array}$ & $\begin{array}{r}24 \cdot 8 \pm 2 \cdot 4 \\
536 \pm 152\end{array}$ & $\begin{array}{l}24 \cdot 9 \pm 2 \cdot 7 \\
892 \pm 156\end{array}$ \\
\hline & Little finger & Middle finger & Thumb & \\
\hline \multicolumn{5}{|c|}{$\begin{array}{l}\text { Group } B: 7 \text { subjects: ulnar deviation of fingers and adduction of thumb } \\
\text { Means and standard deviations of: }\end{array}$} \\
\hline $\begin{array}{l}\text { times of failure (min) } \\
\text { initial strength of movements }(\mathrm{g})\end{array}$ & $\begin{aligned} 25 \cdot 4 & \pm 2 \cdot 3 \\
770 & \pm 216\end{aligned}$ & $\begin{aligned} 25 \cdot 5 & \pm 2 \cdot 4 \\
783 & \pm 180\end{aligned}$ & $\begin{aligned} 25 \cdot 8 & \pm 2 \cdot 7 \\
7921 & \pm 2723\end{aligned}$ & \\
\hline
\end{tabular}


and seven females) underwent definitive experiments in which these complicating factors were, so far as possible, eliminated. Seven of these subjects were taken from Group A, the selection being based solely on availability, and the five new subjects were all given a preliminary trial to establish the probable time of motor failure. No definitive experiments were done with the thumb, since the difficulties of shifting from one transducer to the other were not inconsiderable, and since the definitive results obtained with the finger movements appeared to render further use of the thumb unnecessary.

The order of testing the fingers in each subject was randomized by reference to tables. Thin wooden rods ('orange sticks') to act as splints were fixed to the backs of the fingers with strips of adhesive tape; these kept the fingers satisfactorily straight and did not interfere with deviation movements. Motor testing was initiated two minutes before the time at which the first failure had occurred in the preliminary experiment on that particular subject. This last procedure met with somewhat varying success. It had been hoped that in all subjects the first finger to fail would do so after only one round of contractions. In the event, five subjects behaved according to expectation, but two subjects required two rounds, and three subjects three rounds, of contractions before the first failure occurred. In the remaining two subjects failure of one finger had already occurred by the time testing began, and in these two instances an estimate was made by subtracting half a minute from the time of first testing.

The results, which are shown in Table 2, again show no significant differences in the times of paralysis, and there was also no significant difference between the times recorded in the preliminary and in the definitive experiments (Table 3). The mean strengths of the fingers used for the definitive experiments are also shown in Table 2 , and are all significantly different from each other at the $0.1 \%$ level.

In the definitive, as in the preliminary experiments, a testing round of the four fingers occupied one minute, and it was felt that any differences in the times of failure should not be regarded as meaningful unless they amounted to one minute or more. On this basis the fingers of each subject were ranked in order of failure, ties in the ranking being dealt with by dividing the sum of the appropriate ranks equally between the tying fingers. The individual figures for each finger were then summed to give the best estimate of the true rank (Kendall, 1943). On this basis the little finger obtained 31 ranking points, the ring finger 28 , the middle finger 31 , and the index finger 30. Finally, the differences between the times of failure of the index and the little fingers were noted, since if the paralysis were centripetal these two fingers ought to exhibit the greatest differences. The index finger failed before the little finger seven times, and after it five times; only five of the differences were of one minute or more, and two of these were in favour of the little finger failing first, while the other three were in favour of the index finger.

In two of the definitive experiments a trace (less than $10 \mathrm{~g}$ thrust) of ulnar deviation of the little finger persisted after the failure of the other small muscles. In both cases inspection and palpation showed that this was due to a transmitted pull from the flexor carpi ulnaris, which continued to be active for some time after the abductor digiti minimi had failed.

TABLE 2

DEFINITIVE EXPERIMENTS (12 SUBJECTS: ULNAR DEVIATION OF FINGERS)

\begin{tabular}{lcccc}
\hline & Little finger & Ring finger & Middle finger & Index finger \\
\hline Means and standard deviations of: & & & & \\
times of failure (min) & $25.8 \pm 1 \cdot 8$ & $25 \cdot 2 \pm 2 \cdot 0$ & $25.9 \pm 1 \cdot 5$ & $25 \cdot 5 \pm 1 \cdot 8$ \\
initial strength of movements (g) & $656 \pm 202$ & $377 \pm 135$ & $493 \pm 127$ & $820 \pm 194$ \\
\hline
\end{tabular}

TABLE 3

COMPARISON OF PRELIMINARY AND DEFINITIVE EXPERIMENTS ( 7 SUBJECTS)

\begin{tabular}{|c|c|c|c|c|}
\hline \multirow{2}{*}{ Finger } & \multicolumn{2}{|c|}{ Preliminary } & \multicolumn{2}{|c|}{ Definitive } \\
\hline & $\begin{array}{c}\text { Mean time of failure } \\
(\text { min })\end{array}$ & $\begin{array}{c}\text { Mean contractions before } \\
\text { failure (no.) }\end{array}$ & $\begin{array}{c}\text { Mean time of failure } \\
\text { (min) }\end{array}$ & $\begin{array}{c}\text { Mean contractions before } \\
\text { failure (no.) }\end{array}$ \\
\hline Little & $26 \cdot 8$ & $7 \cdot 1$ & $26 \cdot 1$ & $2 \cdot 7$ \\
\hline Ring & $26 \cdot 3$ & $6 \cdot 3$ & $25 \cdot 5$ & $2 \cdot 1$ \\
\hline Middle & $25 \cdot 7$ & $5 \cdot 4$ & 26.4 & $2 \cdot 7$ \\
\hline Index & $25 \cdot 9$ & $5 \cdot 7$ & $26 \cdot 1$ & $3 \cdot 3$ \\
\hline
\end{tabular}


The interval between failure of the last small muscle and the failure of flexor carpi ulnaris was measured in five subjects, and found to be $3.0 \pm 2.3$ minutes, an interval which is significant at the $5 \%$ level.

EFFECT OF REPEATED CONTRACTIONS Five of the subjects who underwent a definitive experiment also took part in experiments in which the force of ulnar deviation of each finger was measured at one-minute intervals immediately after the inflation of the cuff. The course of failure is shown in the Figure. In order to compensate for possible 'learning' effects, the experiment involving exercise preceded the definitive experiment in two subjects and followed it in three. In all cases the muscles failed more quickly in the experiments involving exercise, and the differences were statistically significant (Table 4); as in the definitive experiments, the individual fingers failed at times which did not differ significantly from each other. No subject complained of discomfort sufficient to make them stop contracting their muscles.

\section{DISCUSSION}

The course of the ulnar nerve in the hand is such that a centripetal paralysis within its territory would show itself in the following order of failure of ulnar deviation: index finger, middle finger, ring finger, little finger. In our experiments there was no indication of such a sequence.

Motor paralysis in experiments involving a compression cuff may result from involvement of either muscle or nerve, or of both. Repeated and rapid contraction of a muscle during asphyxia (a common class exercise in physiology) results in early paralysis, the effect being usually ascribed to the accumulation of metabolites in the muscle. If the muscles are kept inactive, paralysis occurs later, and chemical accumulation in the muscle is probably not a significant factor, since paralysis appears at about the same time if the nerve trunk is directly asphyxiated while the circulation to the muscles is allowed to proceed almost unimpeded (Lewis et al., 1931).

In the present investigation the times of failure in the definitive experiments, in which the muscles were

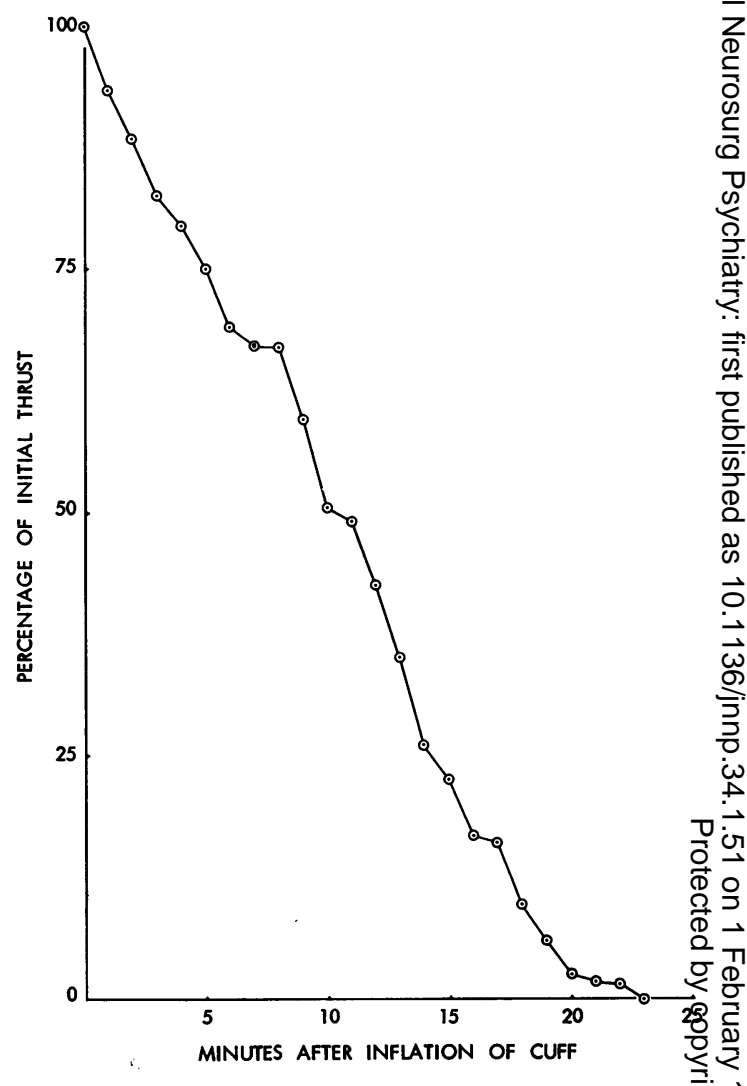

FIG. Time course of motor failure in the hand. The point? on the graph are the means of the readings for all four $\rightarrow$ fingers, expressed as a percentage of the initial mean value.

minimally exercised, did not differ significantly from those in the preliminary experiments, in which a limited amount of exercise usually occurred $\stackrel{\mathbb{D}}{2}$ (Table 3). When regular exercise was undertaken $\overrightarrow{\overrightarrow{0}}$ throughout asphyxia, the muscles failed earlier than 3 they did in either the preliminary or the definitive experiments, and it may be that the earlier onset of paralysis associated with exercise was due to chemical factors affecting the muscle. However, in $\frac{5}{0}$ this case also there was no significant difference

TABLE 4

EFFECT OF EXERCISE ( 5 SUBJECTS)

\begin{tabular}{|c|c|c|c|c|}
\hline & Little finger & Ring finger & Middle finger & Index finger \\
\hline $\begin{array}{l}\text { Mean times of failure with: } \\
\text { minimal number of contractions (min) } \\
\text { repeated contractions at } 1 \text { min intervals (min) } \\
\text { Significance of difference: } P<\end{array}$ & $\begin{array}{l}25 \cdot 4 \\
20 \cdot 9 \\
0.005\end{array}$ & $\begin{array}{c}24 \cdot 9 \\
20 \cdot 3 \\
0 \cdot 01\end{array}$ & $\begin{array}{l}25 \cdot 4 \\
20 \cdot 7 \\
0 \cdot 005\end{array}$ & $\begin{array}{l}24 \cdot 8 \\
20.9 \\
0.02\end{array}$ \\
\hline
\end{tabular}


between the times of failure of the movements of the individual fingers. We may therefore say that, whether the onset of paralysis was due to muscular or nervous involvement, there was no indication of centripetal paralysis within the territory of the ulnar nerve in the hand. The findings therefore lend no support to the theory that the longer motor fibres of the ulnar nerve are more vulnerable to the effects of asphyxia than the shorter fibres, as suggested by Lewis et al. (1931).

It could perhaps be argued that the fibres are indeed affected differentially in this way, but that the effect is masked by the varying strength of the individual muscles involved. Our results would not support this contention. If the weakest muscles tend to fail first, the order of paralysis should be: ring finger, middle finger, little finger, index finger (Tables 1 and 2). To neutralize this supposed tendency the nerve supply to these muscles would have to fail in the order: index finger, little finger, middle finger, ring finger. That is, the longest fibres must fail first, immediately followed by the shortest fibres, and these in turn by the intermediate fibres.

Again, in the preliminary experiments in which adduction of the thumb was used, this movement was roughly ten times as strong as the movements of ulnar deviation of the middle finger and little finger, with which it was compared, yet it failed at the same time. To account for this on the basis of a combination of the factors of initial strength and distance of innervation from the central nervous system it would have to be assumed that there was a very steep gradient of susceptibility between the fibres supplying the first interosseous space and those supplying the third. Yet no such difference could be demonstrated between the fibres supplying the second interosseous space and those supplying the fourth, a distance which is very similar.

A more telling criticism might be that the difference in the lengths of the fibres under test in these experiments was too small to show up any difference between fingers. The mean distance between the point of innervation of the abductor digiti minimi and the point of innervation of the muscles producing ulnar deviation of the index finger is approximately $4 \mathrm{~cm}$ (Sunderland and Hughes, 1946), and this is indeed short compared with the distance between the point of innervation of the abductor digiti minimi and the point at which the first supply to the flexor carpi ulnaris enters the muscle. This distance can be calculated from data provided by Seddon, Medawar, and Smith (1943), and Sunderland and Hughes (1946), and is approximately $25 \mathrm{~cm}$, assuming a forearm length of $25 \mathrm{~cm}$ (Martin, 1928). In the five subjects in whom the point was investigated, the flexor carpi ulnaris remained active for a mean period of three minutes after the time at which the last of the hand muscles tested became paralysed. However, to say that the flexor carpi ulnaris survives the small muscles of the hand is not to say that long nerve fibres are more susceptible to asphyxia than short ones, and other explanations may be available. For example, within the parent trunk of the ulnar nerve the fibres supplying the flexor carpiulnaris tend to lie posteriorly in the region of the medial epicondyle, and it is here that the nerve receives a rich blood supply (Sunderland, 1968) from the anastomosis round the elbow. A certain amount of blood flow through this anastomosis may continue during compression of the arm (Weddell and Sinclair, 1947), and there may be a differential supply to the various groups of nerve fibres.

It can at least be said that any inherent differences between the longer and shorter fibres of the ulnar nerve in their susceptibility to asphyxia do not manifest themselves in differences in the time of failure of observable contractions when the difference in length of the motor fibres concerned is $4 \mathrm{~cm}$ or less. Sinclair (1948) found no difference in the times of extinction of fibres supplying the most proximal and the most distal parts of the sensory distribution of the palmar branch of the ulnar nerve, a distance of about $5 \mathrm{~cm}$.

Both Lewis et al. (1931) and Kugelberg (1948a, b) found that the extensor muscles of the wrist failed before the flexor muscles, and we have confirmed this observation incidentally in two subjects. Any differential susceptibility of the nerve fibres cannot therefore be based, as Lewis et al. (1931) point out, purely on the length of the fibres per se, since the extensor and the flexor muscles lie more or less equidistant from the spinal cord. Clearly other factors, such as the variations in the blood supply and the degree of compression of different nerve trunks, may be decisive.

Motor fibres in the ulnar nerve appear to be somewhat less vulnerable to asphyxia than sensory fibres conveying impulses interpreted centrally as touch, which fail usually between the 20th and the 25 th minute of a compression block (Sinclair, 1948; Sinclair and Hinshaw, 1951). The sensory endpoint is an artificial one, and certainly underestimates the time of complete paralysis of touch sensibility, which is difficult to define (Sinclair, 1948). It may be that the motor endpoint adopted in the present investigation also underestimates the time of complete motor failure. Yet it seems likely that a real difference may exist. Now the length of a sensory fibre supplying the tip of the little finger, measured from the spinal cord, is about the same as that of a motor fibre supplying the adductor pollicis, so the difference 
between sensory and motor fibres, if it is real, has no relation to the lengths of the fibre. The currently accepted explanation is that susceptibility depends on the diameter of the fibres. In experimental asphyxial blocks of the sensory nerves of animals the small myelinated fibres in general tend to fail before the large myelinated fibres, and these in turn before the unmyelinated fibres. In motor nerves the evidence is not only less clear, but sometimes conflicting (Sinclair, 1967). Any interpretation of the differences in behaviour of sensory and motor fibres in human cuff experiments in terms of fibre size is thus open to question.

We should like to thank Professor J. R. Mallard and the staff of the Department of Medical Physics of this university for their design and provision of the transducers used in this investigation, and also all the volunteers who so willingly took part in it, at the cost of some inconvenience and discomfort.

\section{REFERENCES}

Frankenhaeuser, B. (1949). Ischaemic paralysis of a uniform nerve. Acta physiol. scand., 18, 75-98.

Groat, R. A., and Koenig, H. (1946a). Centrifugal deterioration of asphyxiated peripheral nerve. J. Neurophysiol., 9, 275-284.

Groat, R. A., and Koenig, H. (1946b). Centrifugal deterioration of asphyxiated motor nerve within the neural axis. J. Neurophysiol., 9, 463-469.

Kendall, M. G. (1943). The treatment of ties in ranking problems. Biometrika, 33, 239-251.

Kugelberg, E. (1948a). Activation of human nerves by ischaemia. Trousseau's phenomenon in tetany. Arch. Neurol. Psychiat. (Chic.), 60, 140-152.

Kugelberg, E. (1948b). Activation of human nerves by hyperventilation and hypocalcemia. Neurologic mechanism of symptoms of irritation in tetany. Arch. Neurol. Psychiat. (Chic.), 60, 153-164.

Leksell, L. (1945). The action potential and excitatory effects of the small ventral root fibres to skeletal muscle. Acta physiol. scand., Suppl. 31, 1-84.

Lewis, T., Pickering, G. W., and Rothschild, P. (1931). Centripetal paralysis arising out of arrested bloodflow to the limb, including notes on a form of tingling. Heart, 16, 1-32.

Martin, R. (1928). Lehrbuch der Anthropologie, 2nd edition (3 vols.). Fischer: Jena.

Matheson, A. B., Sinclair, D. C., and Skene, W. G. (1970). The range and power of ulnar and radial deviation of the fingers. J. Anat., 107, 439-458.

Seddon, H. J., Medawar, P. B., and Smith, H. (1943). Rate of regeneration of peripheral nerves in man. J. Physiol. (Lond.), 102, 191-215.

Sinclair, D. C. (1948). Observations on sensory paralysis produced by compression of a human limb. J. Neurophysiol., 11, 75-92.

Sinclair, D. C. (1967). Cutaneous Sensation, pp. 306. Oxford University Press: London.

Sinclair, D. C., and Hinshaw, J. R. (1951). Sensory phenomena in experimental nerve block. Quart.J. exp. Psychol., 3, 49-72.

Sunderland, S. (1968). Nerves and Nerve Injuries, pp. $1160_{0}^{\circ}$ i Livingstone: Edinburgh.

Sunderland, S., and Hughes, E. S. R. (1946). Metrical ang non-metrical features of the muscular branches of the ulnar nerve. J. comp. Neurol., 85, 113-126.

Weddell, G., and Sinclair, D. C. (1947). 'Pins and needles observations on some of the sensations aroused in a lim by the application of pressure. J. Neurol. Neurosur Psychiat., 10, 26-46. 